\title{
On the temperature dependence of ferrofluid susceptibility
}

\author{
Yuri Dikansky ${ }^{1}$, Anna Ispiryan ${ }^{1}$, Stanislav Kunikin ${ }^{1, *}$ \\ ${ }^{1}$ North-Caucasus Federal University, Institute of mathematics and natural science, 3550091 Pushkina str., Stavropol, Russia
}

\begin{abstract}
The fulfillment of Curie-Weiss laws for a ferrofluid is critically discussed. The results of investigations of the magnetic susceptibility temperature dependences of ferrofluid and dried powder of magnetic particles are presented. The effective field parameter is computed in approximation of the effective field theory. It is indicated that the value of the effective field parameter is not constant and depends on the concentration of magnetic nanoparticles and the temperature of the sample. It was found that Curie-Weiss law with $\lambda=4 \pi / 3$ is satisfied for ferrofluid with a limited range of volume concentration of magnetic particles.
\end{abstract}

\section{Introduction}

A magnetic colloid, also known as a ferrofluid (FF), is a colloidal suspension of single-domain magnetic particles, with typical dimensions of about $10 \mathrm{~nm}$, dispersed in a liquid carrier [1-3]. The liquid carrier can be polar or nonpolar. Since the nineteen sixties, when these materials were initially synthesized, their technological applications did not stop to increase. As time went by, concurrent to the improvements in the liquids themselves was the discovery of numerous other practical applications for ferrofluids, some of which have gained high commercial importance. At present, ferrofluids are an important class of materials for industrial applications.

First models of ferrofluid magnetization based on the simple idea when magnetic nanoparticles are treated as an ideal paramagnetic gas and magnetic properties were described by Langevin function. At finite concentration, taking into account the magnetic interparticle interaction under the applied field, it is possible to use a mean field approximation, an effective field model, and the magnetization then is described as

$$
H_{\text {eff }}=H+\lambda M \text {, }
$$

where $\lambda$ is the effective field parameter. In the case of the classical dipolar system $\lambda$ is Lorentz parameter that equals $4 \pi / 3$. With the help of Eq. (1) we express equation for initial susceptibility in a form similar to classical Curie-Weiss law:

$$
\chi=\frac{C}{T-T_{\mathrm{C}}},
$$

here $C=\frac{n m^{2}}{3 k}, \quad T_{C}=\lambda C$

The previously conducted experimental studies [4] revealed the limited use of the formula for the effective field in a ferrofluid in the form of expression (2), since it turned out that the effective field parameter does not remain constant when the concentration of colloidal particles changes. At the same time, the temperature studies of the magnetic susceptibility of various samples of ferrofluid at a fixed concentration of the particles showed that at sufficiently high temperatures the CurieWeis law could describe dependence of the susceptibility on temperature. This result indicates the possibility of using the effective field model in this temperature interval.

Actually, well known that some properties of the carrier and magnetic particles strongly depends on temperature. In addition, magnetization relaxation mechanism can switch from Néel to Brownian with temperature change. That means we have to use effective field model more careful. In this paper, we focus on what effects on the magnetic susceptibility has these properties of magnetic particles and carrier.

\section{SAMPLES AND SETUP}

Investigation of the magnetization processes in the ensemble of magnetic nanoparticles, taking into account their interaction, was carried out by an analysis of the temperature dependences of the magnetic susceptibility. The main sample for research was a kerosene based ferrofluid with a concentration of magnetite nanoparticles of 17.6 vol. \%. We changed the concentration by diluting kerosene sample, or by evaporating carrier at $400 \mathrm{~K}$. Additional studies were carried out on a powder containing magnetite nanoparticles. This sample was obtained by complete evaporation of kerosene and further grinding of the dry residue. In this work, the temperature dependences of the magnetic susceptibility obtained on three powder samples were analysed. The difference between these samples was the different size of magnetite nanoparticles.

Investigations of the temperature dependence of the magnetic susceptibility were carried out by the AC-bridge method using a setup described in [5]. The probing field frequency $f$ was in the range from $20 \mathrm{~Hz}$ up to $10^{4} \mathrm{~Hz}$,

\footnotetext{
* Corresponding author: skunikin@yandex.ru
} 
its magnitude $20 \mathrm{~A} / \mathrm{m}$. Experimental data processing take into account effects of carrier's thermal expansion and temperature dependence of the magnetite saturation magnetization..

Table 1. Setting Word's margins.

\begin{tabular}{|c|c|c|}
\hline Sample & Carrier & $\begin{array}{c}\text { Particles } \\
\text { mean } \\
\text { diameter } \\
\text { d, } \mathbf{n m}\end{array}$ \\
\hline 1 & Kerosene & 13.2 \\
\hline 2 & $-/$ powder & 13.2 \\
\hline 3 & $-/$ powder & 9.7 \\
\hline 4 & $-/$ powder & 7.8 \\
\hline
\end{tabular}

\section{RESULTS AND DISCUSSION}

The features of the process of magnetization of ferrofluid are related to the mechanism of relaxation of the magnetic moment of a single-domain colloid particle. Well known that two magnetic relaxation mechanisms are actualized in ferrofluid. The first is the Brownian rotation of particles with a magnetic moment fixed relating to the particle. The second is the rotation of the magnetic moment of the particle, related to the thermal fluctuations the Néel relaxation. The implementation of a specific mechanism is due to both particle size and temperature. The polydispersity of ferrofluids allows one particle to be Brownian, the other to be Néel particles at the same temperature [6]. This leads to the possibility of the appearance of peculiarities in the temperature dependences of the magnetic susceptibility.

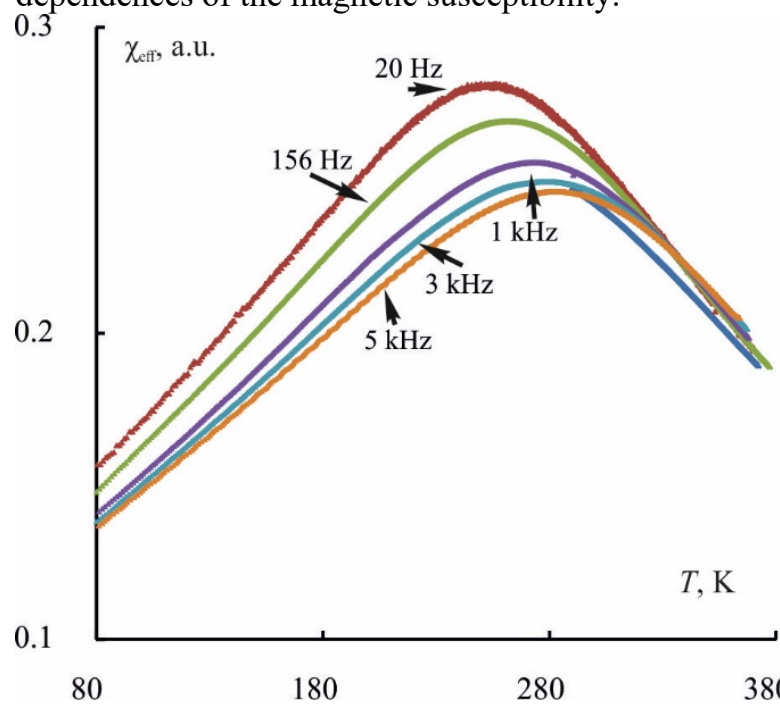

Fig. 1. Temperature dependence of magnetic susceptibility of sample No. 2 at different frequencies of probing field.

For Brownian particles, a change in the viscosity of the carrier can lead to a change in the behaviour of the functional dependencies of the dynamic magnetic susceptibility. For example, when the carrier solidifies, the Brownian degrees of freedom are blocked. With such a phase transition, the magnetic susceptibility should decrease.
An experimentally similar pattern of the dependence of the magnetic susceptibility is described in some papers, for example [7]. For the sample of a ferrofluid No. 1 the maximum of magnetic susceptibility observed at a temperature of $207 \mathrm{~K}$ close to the temperature of solidification of the carrier. Based on the analysis of the temperature dependence, we conclude the Brownian relaxation mechanism for magnetic moments of the particles is dominant for this sample.

The temperature dependence of the magnetic susceptibility of the powders also revealed the peak of the magnetic susceptibility temperature dependence at a specific temperature, depending on the size of the particles and the frequency of the probing field (Fig. 1). In magnetic powders, Brownian degrees of freedom are blocked, and therefore the presence of a maximum unexplainable in the same way as for the sample No. 1. A peak on the temperature dependence of magnetic susceptibility for this sample we associated with the transition of magnetic particles from the superparamagnetic state into ferromagnetic state related to the temperature change. The temperature corresponding to the maximum of the temperature dependence of magnetic susceptibility depends on the frequency of probing field (Fig. 2). Observation of the transition of the ensemble of magnetic particles to a superparamagnetic state is possible if the relaxation time of the ensemble correspond to the observation time (period of probing field) [9]. The relaxation time of the activation magnetization reversal is [10]:

$$
\tau=\tau_{0} \exp \left(\frac{\Delta E}{k T}\right),
$$

here $\tau_{0}=10^{-9}-10^{-10} \mathrm{~s}, \Delta E-$ energy barrier depending on magnetocrystalline anisotropy.

From the Eq. (3), one could obtain that the transition to a superparamagnetic state is observed when:

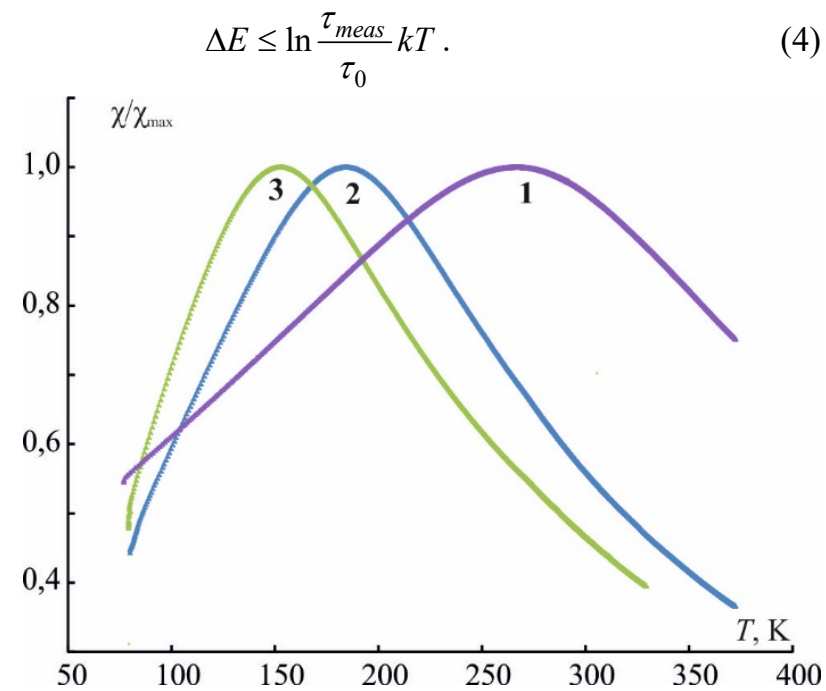

Fig. 2. Normalized temperature dependence of magnetic susceptibility of samples No. 2 (curve 1), No. 3 (curve 2), No. 4 (curve 3). Frequency of probing field $328 \mathrm{~Hz}$.

Sample No. 2 contains larger particles than sample No. 3 and No 4. These leads to increasing of energy barrier magnitude therefore their blocking temperature is higher (Fig. 2). From (4) it appears that the transition of an ensemble of particles into a superparamagnetic state at 
a given temperature is also determined by the observation time (in the case of an AC field, by its period). An increase of the probing field frequency (a decrease in the observation time) leads to a blocking of the direction of the magnetic moment of the particle (transition to the ferromagnetic state) at higher temperatures. This explains the change in the maximum of the magnetic susceptibility temperature dependence with increasing of the probing field frequency.

Simple analysis lead us to figure out that at the temperature corresponding to the peak of the magnetic susceptibility temperature dependence we achieve a situation when $\tau_{\text {meas }}=\tau$. For each curve, we determined temperature corresponding to maxima and also found value $\tau \sim 1 / f$. One could find that the dependence of relaxation time on the temperature of the sample well fitted by Vogel-Fulcher law (Fig. 3)

$$
\tau=\tau_{0} \exp \left(\frac{A}{T-T_{0}}\right) .
$$

Analysis of fitting parameters $\tau_{0}, A$ and $T_{0}$ allows us to compute energy barrier height. For investigated sample, it was about $0.17 \mathrm{eV}$.

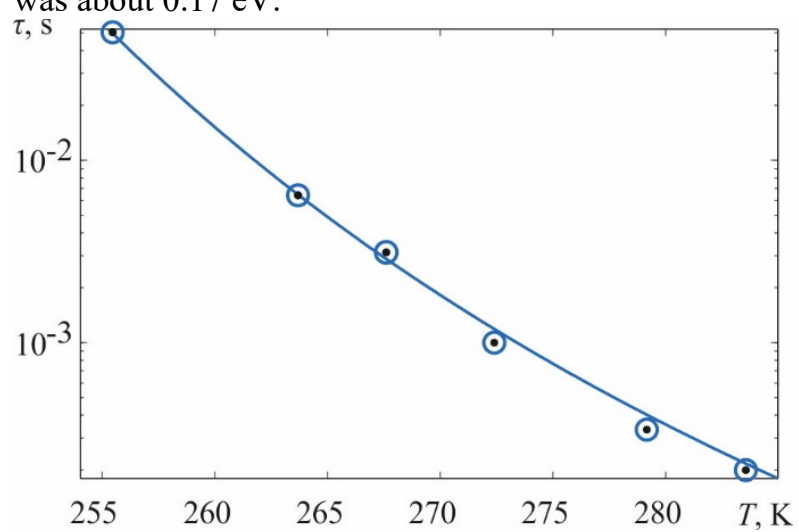

Fig. 3. Dependence of relaxation time computed by temperature dependencies of magnetic susceptibility of sample No. 3 . (Circles - experiment, solid line Vogel-Fulcher law approximation)

Well known that this dependence describes relaxation properties of the Ising-like correlations systems. The results obtained from fitting of experimental data by Vogel-Fulcher law allows us to conclude that relaxation properties of investigated samples are intermediate between those of spin glasses and ensemble of noninteracting ferromagnetic particles. We have figure out that studied sample obeys the behavior similar to a progressive freezing of clusters $[4,8]$.

Thus, investigating the possibility of describing the temperature dependence of the magnetic susceptibility by the Curie-Weiss law we need to exclude the influence of factors associated with different mechanisms of particle's magnetic moment relaxation. To achieve needed conditions we done measurements in the temperature range corresponding to the superparamagnetic state of particles. Superparamagnetic state of investigating samples corresponding to the temperature range higher than the temperature of the peak observed on temperature dependencies of susceptibility at Fig. 1 and Fig. 2.
Therefore, studies of liquid sample No 1 were carried out at temperatures above $310 \mathrm{~K}$, for the magnetic powder with large-sized particles (sample No. 2) - at temperatures above $320 \mathrm{~K}$.

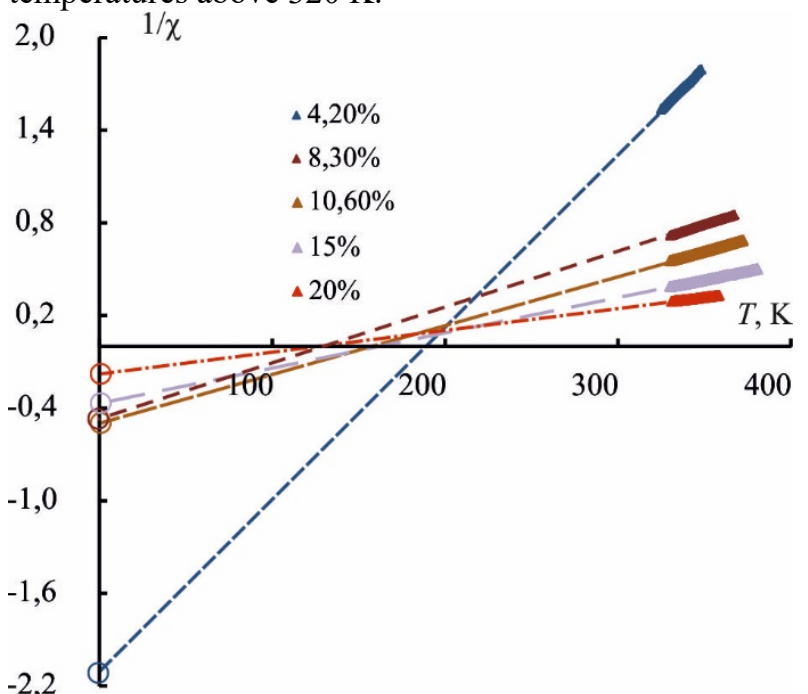

Fig. 4. Temperature dependence of inverse magnetic susceptibility of sample No. 1 at different concentrations of particles at temperatures higher than $310 \mathrm{~K}$ (Solid points experiment, dash and dash-dotted lines - straight-line approximation).

We figured out that the magnetic susceptibility temperature dependence for all the investigated samples of ferrofluid at temperatures above $310 \mathrm{~K}$ is well described by behaviour similar to the classical CurieWeiss law. This allows us to use the theory of the effective field to determine magnetic field acting on the magnetic particle. Figure 4 reveals the dependence of the inverse magnetic susceptibility on the temperature for the sample with different particle concentrations. In this figure, the fitting lines intercept segments of different magnitude on y-axis. Quantitatively the length of these segments are equal to effective field parameter $\lambda$ determined by eq. (2). On the one hand for small concentrations (less than $8 \mathrm{vol} . \%$ ), the magnitude of this parameter exceeds the same value for classical dipolar systems. On the other, an increase in concentration leads to a decrease of $\lambda$ up to zero for more dense samples. This analysis allows us to compute magnitude of $\lambda$ on concentration. Fig 5 . illustrates the dependence of the effective field parameter on the volume concentration of the particles. From the slopes of the straight lines the magnetic moments of the particles were determined. These values are in good agreement with those calculated from the size determined by electron microscopy (Table 1) and a constant intrinsic magnetization. 


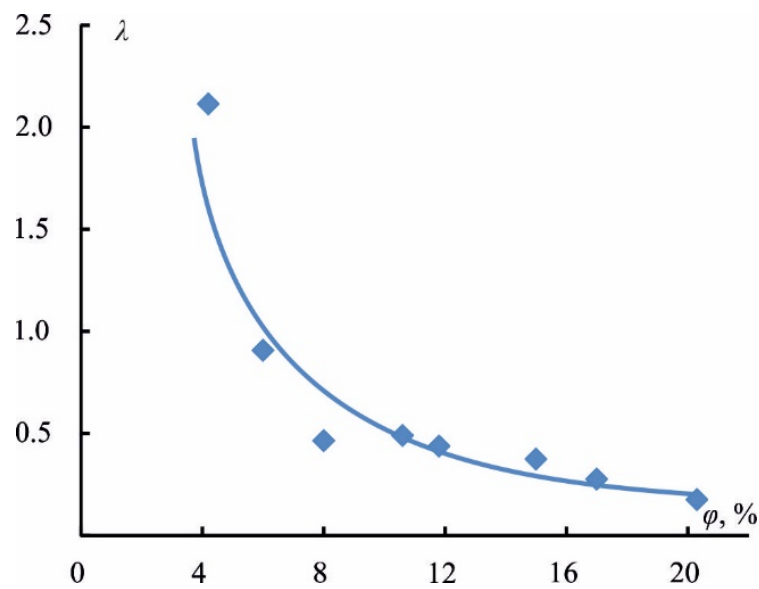

Fig. 5. Dependence of effective field parameter $\lambda$ on concentration of magnetic particles.

With a decrease in the concentration of particles, the intensity of the particle interaction should decrease and for a diluted ferrofluid, the magnetic susceptibility could be expressed by behaviour similar to the Curie law. On the contrary, to predictions experimental results show different behavior. Dependence on Fig. 5 illustrates that while volume concentration of magnetic particles decrease effective field parameter $\lambda$ increase (similar dependence for other samples of ferrofluid were expressed in [9]). This means that intensity of particle interaction increase We assumed that this is due to a violation of the homogeneity of the sample due to the formation of aggregates when the initial sample is diluted by the carrier. In this case, it is possible to form both nanoscale chain aggregates $[10,11]$ and larger ones with non-zero magnetic moments [12-14]. The possibility of its formation was previously presented in [1]. We did not observe aggregates in vivo but found it presence qualitatively by means of the laser correlation spectroscopy and the Vogel-Fulcher behavior analysis. The decrease of $\lambda$ with increasing in volume concentration (especially for the samples corresponding to dense state), is associated with an increase in the impact of the local fields produced by particle's neighbour and contributing for the orientation of the particle moment along the applied field direction.

\section{Conclusion}

Thus, we conclude that the temperature dependence of the magnetic susceptibility of such systems corresponds to the behaviour similars to the Curie-Weiss law at temperature range of 310-380 K. For samples with concentrations of magnetic particles exceeding $8 \%$ the value of the parameter $\lambda$ is always less $4 \pi / 3$, which corresponding to the effective field theory for dipolar systems. It decreases with increasing volume concentration of magnetic particles. With a further decrease in the concentration of particles, the $\lambda$ value, calculated from the temperature dependences of the magnetic susceptibility, increases. It is assumed that this is the result of forming aggregates with uncompensated magnetic moment.

\section{Acknowledgements}

We thank Russian foundation for basic research (project 18-03-00279) and Ministry of Science and Education of Russian Federation for funding.

\section{References}

1. Y. I. Dikansky, D. V. Gladkikh, S. A. Kunikin, and A. A. Zolotukhin, Magnetohydrodynamics 48, 493 (2012).

2. S. Yoon, J. Magn. 16, 368 (2011).

3. Y. I. Dikanskii, V. A. Silaev, K. A. Balabanov, Y. M. Kozlov, and N. G. Polikhronidi, Magnetohydrodynamics 25, 108 (1989).

4. J. L. Dormann, L. Bessais, and D. Fiorani, J. Phys. C Solid State Phys. 21, 2015 (1988).

5. S. A. Kunikin and Y. I. Dikanskii, Tech. Phys. 55, 866 (2010).

6. P. C. Fannin and S. W. Charles, J. Phys. D. Appl. Phys. 22, 187 (1989).

7. A. F. Pshenichnikov, A. V. Lebedev, and K. I. Morozov, Magnetohydrodynamics 23, 31 (1987).

8. V. Kumar, R. Kumar, K. Singh, S. K. Arora, I. V. Shvets, and R. Kumar, J. Appl. Phys. 116, 73903 (2014). 9. Y. I. Dikanskii, A. G. Ispiryan, and S. A. Kunikin, Russ. Phys. J. 60, 181 (2017).

10. A. O. Ivanov, Colloid J. 66, 679 (2004).

11. L. M. Pop and S. Odenbach, J. Phys. Condens. Matter 18, S2785 (2006).

12. D. Eberbeck, A. Lange, and M. Hentschel, J. Appl. Crystallogr. 36, 1069 (2003).

13. A. O. Ivanov and A Y. Zubarev, Phys. Rev. E. Stat. Nonlin. Soft Matter Phys. 64, 41403 (2001).

14. Y. I. Dikansky, K. A. Balabanov, O. V. Borisenko, and V. V. Kiselev, Magnetohydrodynamics 33, 202 (1997). 